\title{
Growth factor analogue shows promise for Rett syndrome in late-stage trial
}

\section{BY PETER HESS}

25 JANUARY 2022

\section{Listen to this story:}

https://www.spectrumnews.org/wp-content/uploads/2022/01/audio-696fc8ef-0c87-4b5ca856-426a568a8163-encodings.mp3

The investigational drug trofinetide, an analogue of insulin-like growth factor 1 (IGF-1), helps ease issues such as anxiety and breathing problems in girls and young women with Rett syndrome, according to unpublished results from a placebo-controlled trial.

Rett syndrome is caused by mutations to MECP2, a gene found on the $\mathrm{X}$ chromosome. The condition affects mostly girls and is characterized by motor issues, breathing and eating problems, anxiety and autism. There are no drugs approved to treat it.

Injections of IGF-1, a protein produced in the liver, repair malfunctioning synapses in mice missing MECP2, according to past research. These mice showed improved breathing and heart function, but not motor skills or coordination. Trofinetide mimics only a portion of IGF-1, and it has been modified to last longer in the body and be administered orally.

In the new trial, 187 girls and young women with Rett syndrome, ranging from 5 to 20 years of age, took twice-daily doses of a liquid containing trofinetide or a placebo for 12 weeks. The 91 participants who took trofinetide showed significantly more improvements in Rett syndrome traits than those on placebo, according to caregiver and physician ratings.

Acadia Pharmaceuticals, the company that sponsored the trial, announced the results in a press release in December. Based on the trial results, Acadia plans to submit a new drug application for 


\section{Spectrum | Autism Research News}

https://www.spectrumnews.org

trofinetide to the U.S. Food and Drug Administration by the middle of 2022, says Deb Kazenelson Deane, senior director of product communications at Acadia.

The magnitude of improvement was small, raising questions about whether the drug's effects are clinically meaningful. But given the dearth of treatments for Rett syndrome, any improvement is important, says Sébastien Jacquemont, associate professor of pediatrics at the University of Montreal in Canada, who was not involved in the study.

"There has been such a small amount of trials that have shown a clear, significant effect, that at this point, as a researcher and as a clinician, I don't really care if it's significant clinically," Jacquemont says. The statistically significant results indicate that researchers have identified a valid treatment target, he says.

The study participants were randomly assigned to the treatment and placebo groups, and the trial was double-blind, so neither the families nor the researchers knew to which group any participant belonged. Parents or caregivers reported their child's progress over the course of the trial using the Rett Syndrome Behavior Questionnaire (RSBQ), which asks about common Rett traits. Clinicians rated the severity of participants' traits on the Clinical Global Impressions Scale.

On both measures, participants who took trofinetide showed more improvement than the placebo group, according to a December presentation by Acadia in an investor webcast. The RSBQ may not be a reliable outcome measure for clinical trials, according to a 2020 analysis. The addition of a clinician-reported scale, however, supports the validity of the RSBQ findings, Deane says.

What remains to be seen is exactly how clinically meaningful these improvements are, says coprincipal investigator Elizabeth Berry-Kravis, professor of pediatrics and neurological sciences at Rush University Medical Center in Chicago, Illinois.

"There are those in the rare disease field, and many families, who would tell you that anything that's an improvement is a clinically meaningful improvement because we have nothing that's specifically targeted to our disease," she says.

Because the Food and Drug Administration is increasingly stringent about ensuring that clinical trials yield results that are not only statistically significant but also clinically meaningful, it's possible that Acadia's discussions with the regulator will result in requirements to that end, BerryKravis says.

But future Rett therapies will probably include some combination of drugs or other therapies, Jacquemont says. "I don't think any treatment alone would have an effect of clinical significance."

Cite this article: https://doi.org/10.53053/BMOS2862 